\title{
The Determination of Caffeine Level of Wolaita Zone, Ethiopia Coffee Using UV-visible Spectrophotometer
}

\author{
Zewdu Tadesse Wondimkun ${ }^{1,}$, , Abdisa Gebisa Jebessa ${ }^{1}$, Liboro Hundito Molloro ${ }^{1}$, Tesfaye Haile ${ }^{2}$ \\ ${ }^{1}$ Department of Chemistry, College of Natural and Computational Science, Debre Berhan University, Debre Berhan, Ethiopia \\ ${ }^{2}$ Department of Chemistry, College of Natural and Computational Science, Wolaita Sodo University, Wolaita Sodo, Ethiopia
}

Email address:

Zewdub23@yahoo.com (Z. T. Wondimkun)

${ }^{*}$ Corresponding author

\section{To cite this article:}

Zewdu Tadesse Wondimkun, Abdisa Gebisa Jebessa, Liboro Hundito Molloro, Tesfaye Haile. The Determination of Caffeine Level of Wolaita Zone, Ethiopia Coffee Using UV-visible Spectrophotometer. American Journal of Applied Chemistry.

Vol. 4, No. 2, 2016, pp. 59-63. doi: 10.11648/j.ajac.20160402.14

Received: February 15, 2016; Accepted: February 24, 2016; Published: March 23, 2016

\begin{abstract}
Coffee is an important commodity culturally, commercially and economically in the world. Because of its consumption in most countries in the world, it is important to investigate the exact amounts of its chemical constituents. Caffeine is one of the main components of coffee that affect the quality of coffee. In this research, rapid, simple, accurate, reproducible and cheap UV-Vis spectrometer was used for the quantification of caffeine in coffee and dichloromethane was used as a solvent for the coffee samples taken from Wolaita zone of five woredas. The results of this research show that the amount of caffeine of the coffee samples ranged from $(450.13$ to $579.60(\mathrm{mg} / \mathrm{L})$. The amounts of caffeine in Bolso Bomba coffee is the greatest, of all the other coffee samples studied in Wolaita Zone. On the other hand, Kindo Koisha and that of Bolso sore coffee has the least caffeine content compared to the other coffee samples measured. The caffeine content of Sodo Zuria coffee and Humbo coffee samples has an average amounts of caffeine.
\end{abstract}

Keywords: Coffee, Caffeine, UV-visible Spectrophotometer

\section{Introduction}

Coffee is one of the most popular beverages in the world and the most traded commodity second after oil. Arabica coffee is more economical and it represent three quarters of the world coffee productions. Ethiopia is the center of origin and genetic diversity for Arabica coffee. Coffee had been and still contributes the lion share in the national economy being the leading source of foreign exchange earnings. It is an essential commodity to the livelihood of millions of Ethiopians. The major coffee producing regions in the country are Oromiya and Southern Nations Nationalities and Peoples Region. Whereas other region like Tigray produces coffee in small amount [1-3]

Ethiopia is the largest country producing Arabica coffee and an original home of coffee along with the highest diversity in its genetic resource. Coffee plant was originally found and cultivated in Kafa province of Ethiopia from which it got its name around 1000 A.D. Arab people took the coffee seeds from this region and started the first coffee plantation. Then it spread to the whole Europe [4, 5].

Coffee quality has critical importance to the coffee industry. It is defined differently in each stage of its production. Quality is a product that has desirable clean raw and roasted appearance, attractive flavor, good cup taste (balanced acidity and body) and level of caffeine. Factors that determine coffee quality are numerous. The genotype, climatic conditions, soil characteristics, agricultural practices, harvesting methods and post-harvest processing techniques are the major attributes for deterioration of coffee quality $[1,4,6]$.

Caffeine is a naturally occurring substance found in the leaves, seeds and/or fruits of at least 63 plant species worldwide and is part of a group of compounds known as methylxanthines. 
<smiles>Cn1c(=O)c2c(ncn2C)n(C)c1=O</smiles>

Scheme 1. The chemical structure of Caffeine (1, 3, 5-trimethylxanthine)

The most commonly known sources of caffeine are coffee, cocoa beans, kola nuts and tea leaves. The amount of caffeine in food products varies depending upon the serving size, the type of product, and preparation method. With teas and coffees, the plant variety also affects the caffeine content. Caffeine's popularity stems mainly from the fact that it is a pharmacologically active substance and a mild central nervous system stimulant. That is why many students drink coffee or soda to help them feel alert [7, 8].

Many consumers prefer to avoid caffeine partially or altogether, due to its stimulant effects and others, still on health concern. This makes decaffeination of tea an important industrial process. In addition, caffeine has a slightly bitter flavor. As a result, decaffeinating coffee beans and tea leaves will leave the flavor slightly changed, even if no other components are lost. It should be noted that, decaffeinated coffee and tea are not caffeine free [6,7].

There are many sources of caffeine available on the market today. In addition to being found in coffee, tea, energy waters, cocoa and carbonated beverages, caffeine is also found in a variety of chocolates. It is also present in a number of weight loss supplements in the form of kola nut seeds, bissey nut, guarana, citrus aurantlum extract and yerba mate. It is important to know what form of caffeine is in any diet product you purchase as some do come with safety warnings. It is also important to avoid mixing too many forms of caffeine in one formula as this can lead to restlessness, sleeplessness and heart irregularities. Below is a list of the caffeine content of some foods [9].

Table 1. Caffeine content of some foods of different varieties.

\begin{tabular}{lllll}
\hline Sources of caffeine & Serving size & Caffeine $(\mathbf{m g})$ & Caffeine $(\mathbf{m g} / \mathbf{m l})$ & Caffeine $(\mathbf{m g} / \mathbf{L})$ \\
\hline Coffee & $300 \mathrm{ml}(10 \mathrm{oZ})$ & $142-214$ & $0.47333-0.71333$ & $473.33-713.33$ \\
Chocolate milk & $250 \mathrm{ml}(8 \mathrm{oZ})$ & $5-8$ & $0.02-0.032$ & $20-32$ \\
Cola soft drink & $355 \mathrm{ml}(12 \mathrm{oZ})$ & $36-46$ & $0.10140-0.12957$ & $101.40-129.57$ \\
Iced tea & $300 \mathrm{ml}(10 \mathrm{oZ})$ & 30 & 0.1 & 100 \\
Decaffeinated coffee & $300 \mathrm{ml}(10 \mathrm{oZ})$ & $5-10$ & $0.01666-0.03333$ & $16.66-33.33$ \\
\hline
\end{tabular}

Ref: The Public Health Nutritionists of Saskatchewan 2010

Like many conjugated organic molecules, caffeine absorbs radiation with a wavelength around $260 \mathrm{~nm}$. A conjugated system is one containing two double bonds separated by a single bond. This conjugated pattern may be repeated several times in the molecule. If a series of caffeine standards are analyzed in this region of absorption and a Beer's law is plot prepared, then the amount of caffeine in another substance can be determined $[7,10]$.

Caffeine also stimulates the stomach to pour out large amounts of acid. This in turn leads to burning in the pits of the stomach and aggravates peptic ulcers of the stomach and duodenum. It also raises blood sugar level as a result of quickening of respiration. It also reduces blood flow to the brain by causing the brains blood vessels to constrict. It also may induce benign (noncancerous) breast diseases and may worsen pre-menstrual symptoms in women who overuse it $[6,11]$.

Caffeine crosses the placenta and enters the fetal circulation and its use at a pharmacological level has been associated with low birth weight. Excessive consumption during lactation may cause irritability and wakefulness in a breast - fed baby $[7,12,13]$.

Caffeine has a mild analeptic (respiratory stimulating activity) effect. Other action includes cardiac stimulation which may produce tachycardia dilation of coronary and peripheral blood vessels, constriction of blood vessels and skeletal muscles. It increases the risk of spontaneous abortion in women. An excessive intake of caffeine in some persons appears to augment the sensitivity of the heart to emotional and other factors and so increase the incidence of extra systoles and other arrhythmias. Since caffeine affect the central nervous system conversely, omission of a habitual morning dosage often results in nervousness irritability, drowsiness, poor work performance and headache curable only by taking more caffeine [4, 14-16].

Generally it is important to collect precise information of their caffeine content of different coffee Varity, due to the wide spread consumption of caffeine and its health impact were the main reason to conduct this research.

\subsection{Methods Used for the Determination of Caffeine}

A wide variety of methods have been employed to analyze and quantify the caffeine content of coffee, some common beverages and soda drink such as high performance liquid chromatography (HPLC), UV spectroscopy, Fourier Transform Infrared spectroscopy, Ion chromatography, liquid chromatography coupled to mass spectrometry and Nuclear magnetic resonance (NMR) spectroscopy [11].

Most research activities have been focused on chromatographic methods; however, spectrophotometric determination is preferred because of its rapidity, high accuracy and reproducibility. Moreover, HPLC is an expensive and resource-hungry technique that is not typically found in the scientific teaching labs of schools and colleges. UV/Vis spectroscopy is routinely used in analytical 
chemistry for the quantitative determination of different analytes, such as transition metal ions, highly conjugated organic compounds, and biological macromolecules. Spectroscopic analysis is commonly carried out in solutions but solids and gases may also be studied $[8,17]$.

\subsection{Coffee Productions in Wolaita Zone}

Wolaita zone is one of the thirteen zones of Southern Nations Nationalities and Peoples Region covering an area of $4,471.3 \mathrm{~km}^{2}$. Topographically the zone lies on an elevation ranging from 1,200 to 2.950 meters above sea level. The total population of the zone is estimated about 1,721,339 with a density of 385 inhabitants per square kilometer. The zone has three agro -ecological zones: Dega (3\%), Weynadega $(57.96 \%)$ and Kolla (40\%). The annual average temperature of the zone is $15.1{ }^{\circ} \mathrm{C}$ and the mean annual rainfall ranges from 1,200 to $1,300 \mathrm{~mm}$. Regarding the land utilization data, 261,000 hectares (ha) is used for cultivation, 5,318 ha for grazing, 8,261 ha. Bush- land and the remaining 35,382.5 ha is a cultivable land. From the cultivated land 9,180.64 ha were the share of coffee.

\section{Materials and Methods}

\subsection{Description of Study Area}

The Coffee samples were collected / purchased from Wolaita Zone of five different woredas, namely Boloso sore, Boloso Bombe, KindoKoisha, SodoZuria, and Humbo of model farmers. Those areas are selected purposefully because of two reasons. The first reason is due to their productivity and the other is due to their geographical location.

\subsection{Materials and Chemicals}

UV-Visible Spectrophotometer (18-1884-01-0076, spectral band width $2.00 \mathrm{~nm}$ ), $1 \mathrm{~cm}$ quartz cuvettes, droppers, ring stands, vacuum pump, Buchner funnel, filter paper: $0.7 \mathrm{~cm}$, iron rings, separatory funnel 125,250 and $500 \mathrm{ml}, 50 \mathrm{ml}$ graduated cylinders, Erlenmeyer flasks, pipettes , round bottom flask $250 \mathrm{ml}, 24 / 29$ neck size, $25,50 \mathrm{ml}, 100 \mathrm{ml}$ and $250 \mathrm{ml}$ of dried volumetric flask. The following chemicals were used: standards caffeine powder, chloroform, grinds coffee, sodium carbonate $\left(\mathrm{Na}_{2} \mathrm{CO}_{3}\right)$, distilled water, dichloromethane $\left(\mathrm{CH}_{2} \mathrm{Cl}_{2}\right)$, and sodium sulphate $\left(\mathrm{Na}_{2} \mathrm{SO}_{4}\right)$.

\subsection{Preparation of Standard Solution}

A 1000ppm stock standard of caffeine was prepared by dissolving $200 \mathrm{mg}$ of standard caffeine powder in $200.0 \mathrm{ml}$ distilled water. Working standards were prepared by pipetting $25,12.5,10,7.5,5$ and $2.5 \mathrm{ml}$ aliquots of the stock standard solution into separate $50.0 \mathrm{ml}$ volumetric flasks and diluting to volume with purified water and stirred by magnetic stirrer for $30 \mathrm{~min}$. The absorbance of each solution was measured using a UV/Vis spectrophotometer at absorption maximum of $260 \mathrm{~nm}$. The absorbance values were then plotted against concentrations to generate a calibration curve [17].

\subsection{Caffeine Extraction Procedure}

A $50 \mathrm{ml}$ aliquot was taken from each working standard or sample solution. This aliquot was placed into a separating funnel and $25 \mathrm{ml}$ of dichloromethane was added. The caffeine was extracted by inverting the funnel at least three times, venting the funnel after each inversion. The dichloromethane layer was removed to a clean flask and the extraction procedure was repeated twice more and the solvent layers combined [17].

\subsection{Sample Preparation}

$100 \mathrm{mg}$ of dried and grind sample was mixed with $100 \mathrm{mg}$ of magnesium oxide and $1 \mathrm{~mL}$ distilled water in a flask. The mixture was heated on steam bath for $20 \mathrm{~min}$. To the residue, 5 $\mathrm{mL}$ chloroform was added and extracted by sonication for 15 min and anhydrous sodium sulphate was added to it. The mixture was filtered through filter paper (Whatman No. 1) and washed three times with $5 \mathrm{~mL}$ dichloromethane. The final volume was adjusted to $15 \mathrm{~mL}$ with dichloromethane [17].

\subsection{Quantitative Determination of Caffeine}

Quantitative analysis of caffeine was performed by a UV/Vis Spectrophotometer. The $\lambda_{\max }$ was determined by scanning the standard solution from 200-600 $\mathrm{nm}$ and the obtained results gave an absorption spectrum, which was characterized by a single intensive absorption band located in the UV range at $\lambda_{\max }=260 \mathrm{~nm}$. Standard linear calibration curve was run to obtain the linear range of sample analysis, correlation factor was with accepted value $>(0.996)$ and the standard calibration curve was linear over the range (10-50) ppm caffeine. The quantitative amount of caffeine in samples (ppm) was then determined using the standard curve [17].

\section{Results and Discussion}

\subsection{Results}

The calibration graph correlating to the absorbance and concentration of pure caffeine in chloromethane was constructed at the highest peak for concentration range. Figure 1 indicates that absorbance is directly proportional to concentration or in other words, Beer-Lambert law is valid.

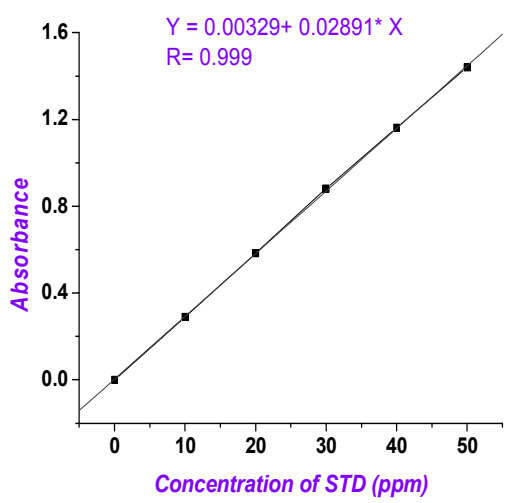

Fig. 1. Calibration curve for STD caffeine concentration. 
A linear regression of concentration vs. absorbance allowed the factor of 34.5 , included in equation 1 , to be determined. Equation lwas then used to calculate the concentration of caffeine in the extracted sample solution, from the solutions measured absorbance value.

$$
\text { Conc }(\text { ppm })=34.5 x \mathrm{Abs}
$$

Table 2. The caffeine concentration of the extracted sample

\begin{tabular}{lll}
\hline Sample & Absorbance & Concentration of Extracted Sample (ppm) \\
\hline A & 0.98 & 33.81 \\
B & 1.09 & 37.60 \\
C & 0.97 & 33.46 \\
D & 1.07 & 36.91 \\
E & 1.02 & 35.19 \\
\hline
\end{tabular}

The experimental result for the determination the amounts of caffeine in five samples of Wolaita Zone obtained from spectrophotometer are described in Table 2 and for convenience, we used to represent coffee sample by symbols in Table 2. The representations are as follows, Bolso Sore coffee (A), Boloso Bomba coffee (B), Kindo Koisha coffee (C), Sodo Zuria coffee (D) and Humbo coffee (E).

The final caffeine content of the beverage under test is then calculated from the extracted sample solution's concentration using equation 2. Dividing this value by the volume of the drink gives the caffeine content per $\mathrm{ml}$ [17].

$$
\text { Caffeine content }(\mathrm{mg})=\frac{\text { Conc. }(\text { ppm }) *(\text { Totalsamplevolume }(\mathrm{ml}))^{2}}{\text { Measuredsamplevolume }(\mathrm{ml}) * 1000)}(2)
$$

Table 3. The calculated caffeine content of the sample coffee.

\begin{tabular}{lllll}
\hline Sample & Total Sample volume $(\mathbf{m l})$ & $\begin{array}{l}\text { Measured sample } \\
\text { Volume(ml) }\end{array}$ & $\begin{array}{l}\text { Caffeine Content Per } \\
\text { Serving }(\mathbf{m g})\end{array}$ & $\begin{array}{l}\text { Caffeine Content } \\
(\mathbf{m g} / \mathbf{m l})\end{array}$ \\
\hline A & 150 & 10 & 76.07 & $\begin{array}{l}\text { Caffeine Content } \\
(\mathbf{m g} / \mathbf{L})\end{array}$ \\
B & 150 & 10 & 84.61 & 0.50715 \\
C & 150 & 10 & 75.29 & 0.56407 \\
D & 150 & 10 & 83.05 & 0.50197 \\
E & 150 & 10 & 79.17 & 0.55366 \\
\hline
\end{tabular}

The results of this research show that the amount of caffeine of the coffee samples ranged from 501.97 to 564.07 $(\mathrm{mg} / \mathrm{L})$. The level of caffeine in all five coffee samples studied in Wolaita zones are almost in the same range. However, the amounts of caffeine in Bolso Bomba coffee is the greatest, of all the other coffee samples studied in Wolaita Zone. On the other hand, Kindo Koisha and that of Bolso sore coffee has the least caffeine content compared to the other coffee samples studied. As can be seen from the Table 3, caffeine content of Sodo Zuria coffee and Humbo coffee samples has an average amounts of caffeine.

\subsection{Discussion of Results}

The results of this analysis show that the amount of caffeine in all five samples ranges from 501.97 to 564.07 $(\mathrm{mg} / \mathrm{L})$ were in an average amounts compared to the literature value of different varieties of coffee (473.33 $713.33 \mathrm{mg} / \mathrm{L}$ ) and which is very good because mild amounts caffeine is advised for health and even the amounts of caffeine is in excess or extra-large it may needs decaffeination to avoid the excess caffeine from the coffee.

The level of caffeine in all five coffee samples studied in Wolaita zones are almost in the same range. However, the level of caffeine in Bolso Bomba coffee is the greatest, of all the other coffee samples studied in Wolaita Zone. On the other hand, Kindo Koisha and that of Bolso sore coffee has the least caffeine content compared to the other coffee samples studied. As can be seen from the Table 3, caffeine content of Sodo Zuria coffee and Humbo coffee samples has an average amounts of caffeine. This small variation of the level of caffeine in the five coffee samples studied in Wolaita Zone may be due to different reasons: most commonly it is because of the roasting processes, dark-roast coffee may contain less caffeine than the lighter product because roasting reduces the caffeine content of the coffee bean (Lovett, 2005), the other reason may be due to their varieties (climatic conditions, soil characteristics, agricultural practices, harvesting methods and post-harvest processing techniques are the major attributes), and the last but not the least may be due to the matrix effect of UV absorption.

\section{Conclusion and Recommendation}

\subsection{Conclusion}

Due to the wide spread consumption of caffeine, it is important to collect precise information of their content in foods. Most research activities have been focused on chromatographic methods; however, spectrophotometric determination is preferred because of its rapidity, high accuracy and reproducibility. Furthermore, UV-visible spectrophotometer is cheap and it is found in many laboratories. Many consumers prefer to avoid caffeine partially or altogether, due to its stimulant effects and others, still on health concern. This makes decaffeination of coffee an important industrial process. In addition, caffeine has a slightly bitter flavor. As a result, decaffeinating coffee beans and tea leaves will leave the flavor slightly changed, even if no other components are lost. It should be noted that, decaffeinated coffee and tea are not caffeine free.

The coffee studied in all five woredas of Wolaita Zone as indicated has mild or averages caffeine contents compared to the literature value of different varieties coffee. Although, as ECX (Ethiopia Coffee Commodity Exchange) of Wolaita branch described that the coffee has attractive flavor, good cup test and good stimulant power. Moreover, it is purely organic coffee and it is one of the highest commercial crops in the area. Adding this all together, Wolaita zone coffee has good quality in both cup taste and caffeine level. So that it 
should have its own brand in the coffee market to that of Jimma and Sidama.

\subsection{Recommendation}

Wolaita Zone has large coffee production as the statistic obtained from the agricultural office has shown. The coffee taste as witnessed from the "Jebena Buna" of the area and the Wolaita Sodo ECX office is very special having comparably mild amount of caffeine as the output of this research has shown. Till the completion of this research paper, the foreign exchange process of the coffee of this area is under SidamaC. The coffee producing farmers are using organic fertilizer or compost. Good coffee is being produced in this area. The concerned bodies should give more attention to this area to promote the current status of the coffee as Wolaita Zone will be one of the country's main sources of foreign exchange income regarding Coffee.

\section{Acknowledgements}

This work was funded by Wolaita Sodo University. The authors are thankful to JJ Labo Glass PLC for their cooperation to use their instrument in the preparation, extraction and determination of the level of caffeine from each coffee sample.

\section{References}

[1] Taye K., Ashenafi A., Alemseged Y., Teshome K., \& Wondiyfraw T. The contribution of coffee research for coffee seed development in Ethiopia. Journal of Agricultural Research and Development, 2011; 1(1), 009-016.

[2] Nebesny, E., \& Grazyna, B., Evaluation of sensory attributes of coffee brews from Robusta coffee roasted under different conditions. Eur. Food Res, 2006; 224, 159-165.

[3] Abrar S., \& Nugussie M., Evaluation of coffee quality of Tigray Regional State, Ethiopia (Case study Raya Azebo District). Prudence Journal of Agricultural Research, 2013; 1(2), 3-6.

[4] Behailu W., Abrar S., Nugussie M., \&Solomon E., Coffee processing and quality research in Ethiopia, 2008.
[5] Wanyika H. N., Gatebe E. G., Gitu L. M., Ngumba E. K., \& Maritim C. W. Determination of caffeine content of tea and instant coffee brands found in the Kenyan market African. Journal of Food Science, 2010; 4(6), 353-358.

[6] Ogah C. O., \& Obebe O.T, Caffeine content of cocoa and coffee beverages in Lagos, Nigeria. Journal of innovative research in engineering and sciences, 2012; 3(1), 404-411.

[7] Kapil K., Sumit K., \& Yoti M., Estimation of caffeine in different beverages by Ultraviolet Spectroscopy. Int. J. of Pharm. \& Life Sci. (IJPLS), 2011; 2(11), 1214-1215.

[8] Forshed J., Erlandsson B., \& Jacobsson P., Quantification of aldehydes impurities in poloxamer by $1 \mathrm{H}$ NMR spectroscopy. Analytica Chimica Acta, 2005; 552, 160-165.

[9] Tadelech A., \& Gholap A. V., Characterization of caffeine and determination of caffeine in tea leave using Uv-visible spectrometer. African Journal of Pure and Applied Chemistry. 2011; 5(1), 1-8.

[10] Kalra K., Kumar S., \& Maithani J., Estimation of Caffeine in different beverages by Ultraviolet Spectroscopy. International Journal of Pharmacy and Life Sciences, 2011; 2 (11), 12141215.

[11] Smith AP., Caffeine at Work. Hum Psychopharmacology, 2005; 20(6), 441.

[12] Rogers P., \& Dernoncourt C., Regular caffeine consumption: a balance of adverse and beneficial effects for mood and psychomotor performance. Pharmacol Biochem Behav, 1998; 59(4), 1039-45.

[13] Stanley D., Passmore R., \& Brock J., Human nutrition and dietetics. Longman publishers, 1979; 2 (11): 200.

[14] Mei M., Mohammed I., Badawi A., \& Abdalla A., Determination of Caffeine in Some Sudanese Beverages by High Performance Liquid Chromatography. Pakistan Journal of Nutrition, 2012; 11 (4), 336-342.

[15] Thompson R., \& Keene K., The pros and cons of caffeine. The Psychologist (The British Psychological Society), 2004; 17 (12), 698-701.

[16] Bibby scientific. The quantitative determination of caffeine in beverages and soft drinks using UV wavelength spectroscopy.jenwayhelp@bibby-scientific.com. www.jenway.com 